\title{
High prevalence of herpes simplex virus (HSV)- type 2 co-infection among HIV- positive women in Ukraine, but no increased HIV mother-to-child transmission risk
}

Karoline Aebi-Popp ${ }^{1 *}$, Heather Bailey ${ }^{2}$, Ruslan Malyuta ${ }^{3}$, Alla Volokha ${ }^{4}$, Claire Thorne ${ }^{2}$ and The Ukraine European Collaborative Study in EuroCoord

\begin{abstract}
Background: Over 3500 HIV-positive women give birth annually in Ukraine, a setting with high prevalence of sexually transmitted infections. Herpes simplex virus Type 2 (HSV-2) co-infection may increase HIV mother-to-child transmission (MTCT) risk. We explored factors associated with HSV-2 seropositivity among HIV-positive women in Ukraine, and its impact on HIV MTCT.

Methods: Data on 1513 HIV-positive women enrolled in the Ukraine European Collaborative Study from 2007 to 2012 were analysed. Poisson and logistic regression models respectively were fit to investigate factors associated with HSV-2 seropositivity and HIV MTCT.
\end{abstract}

Results: Median maternal age was 27 years (IQR 24-31), 53 \% (796/1513) had been diagnosed with HIV during their most recent pregnancy and $20 \%$ had a history of injecting drugs. Median antenatal CD4 count was $430 \mathrm{cell} / \mathrm{s} / \mathrm{mm}^{3}$ (IQR 290-580). Ninety-six percent had received antiretroviral therapy antenatally. HSV-2 seroprevalence was $68 \%$ (1026/1513). In adjusted analyses, factors associated with HSV-2 antibodies were history of pregnancy termination (APR 1.30 (95 \% Cl 1.18-1.43) for $\geq 2$ vs. 0), having an HIV-positive partner (APR 1.15 (95\% Cl 1.05-1.26) vs partner's HIV status unknown) and HCV seropositivity (APR 1.23 (95\% Cl 1.13-1.35)). The overall HIV MTCT rate was $2.80 \%$ (95\% Cl 1.98-3.84); no increased HIV MTCT risk was detected among HSV-2 seropositive women after adjusting for known risk factors (AOR 1.43 (95\% Cl 0.54-3.77).

Conclusion: No increased risk of HIV MTCT was detected among the $68 \%$ of HIV-positive women with antibodies to HSV-2, in this population with an overall HIV MTCT rate of $2.8 \%$. Markers of ongoing sexual risk among HIV-positive HSV-2 seronegative women indicate the importance of interventions to prevent primary HSV-2 infection during pregnancy in this high-risk group.

Keywords: HIV, Pregnancy, Herpes simplex virus, Mother to child transmission

\footnotetext{
* Correspondence: mail@aebi-popp.com

'Division of Infectious Diseases, University Hospital Bern, CH-3010 Bern,

Switzerland

Full list of author information is available at the end of the article
} 


\section{Background}

In Ukraine HIV prevalence among pregnant women was $0.8-1.0 \%$ between 2009 and 2013, and over 3500 HIV-positive pregnant women gave birth in 2013 [1]. Initially injecting drug use drove the HIV epidemic in Eastern Europe [2] but the main mode of acquisition shifted to heterosexual transmission in 2008 [1] and $45 \%$ of new HIV infections in 2013 were in women. After the collapse of the Soviet Union, social changes and poor public health provision resulted in an epidemic of sexually transmitted infections (STIs), particularly syphilis. Although syphilis rates subsequently declined, rates of other STIs remain higher in Eastern than Western Europe [3]; in a study of pregnant HIVpositive women delivering in 1999-2005, those in Ukraine had a 10-fold higher probability of having syphilis, chlamydia or Trichomonas vaginalis than their counterparts in Western Europe [4].

Prevalence of Herpes simplex virus Type 2 (HSV-2) infection, the leading cause of genital ulcer disease worldwide, is around 17-22 \% in adults across Europe and the United States with higher prevalence in HIVpositive individuals (35-40 \%) [5]. It is well established that genital ulcer disease caused by HSV-2 increases risk of HIV sexual acquisition [6]. During latency HSV-2 is able to avoid clearance by the immune system, but laboratory tests show specific antibodies and it can cause recurrent activations, which are more common and of longer duration with HIV co-infection [7, 8]. This synergistic relationship between HIV and HSV-2 also results in more frequent subclinical episodes of HSV-2 reactivation in HIV-positive individuals, which seems to be associated with increases in plasma and genital tract HIV viral load [9].

Mother-to-child-transmission (MTCT) rates of HIV around $0.5 \%$ have now been reported from Western Europe [10, 11]. In Ukraine, the prevention of MTCT (PMTCT) programme has achieved considerable success, with MTCT rates reduced from $15 \%$ in 2001 to $7 \%$ in 2007 and around $4 \%$ in 2010, following introduction of WHO Option B from 2007 [12, 13] (i.e. combined antiretroviral therapy for all HIV-positive pregnant women). Recent national reporting from Ukraine indicated a MTCT rate of $4.3 \%$ in 2012 [1]. Some data suggest an increased risk of HIV MTCT for HSV-2/HIV coinfected women in general [14] or with genital ulcers [15] and a several trials showed decreased HIV RNA levels in plasma or breastmilk after treatment with Valacyclovir $[16,17]$.

Our primary aim was to investigate the influence of HSV-2 co-infection on HIV MTCT risk in Ukraine. Secondary aims were to describe the prevalence of HSV-2 antibodies among HIV-infected childbearing women and associated risk factors.

\section{Methods}

\section{Study setting and subjects}

The European Collaborative Study (ECS) is a consented cohort study of HIV-positive pregnant women and their infants, which has enrolled women at regional HIV/AIDS centres in Ukraine since 2000. Women diagnosed with HIV before or during pregnancy or intrapartum and delivering a live-born infant are eligible. Linked anonymous data on maternal, delivery and infant characteristics are collected on standard questionnaires [13]. Between 2007 and 2012, HIV-positive women were enrolled at five centres (Odessa, Kyiv, Donetsk, Mykolaiv and Krivoy Rog) into a nested postnatal sub-study, normally within 3-6 months of delivery, with the aim of obtaining longitudinal information following delivery. Postnatal women self-reported information on socio-demographic characteristics and health behaviours (including drug use, access to contraception and HIV status of current partner) while clinicians provided clinical information [18]. Ethical approval has been obtained from the Great Ormond Street Hospital for Children NHS Trust/Institute of Child Health Research Ethics Committee (reference 96EB02). We obtained also local approvals from all the participating HIV/AIDS Centres.

The study population for this analysis was 1513 women and their infants enrolled in the Ukraine ECS since 2007, with linked data from the postnatal cohort on maternal HSV-2 serostatus.

\section{Definitions}

Injecting drug use (IDU) history was defined as selfreport, clinician's assessment, or neonatal abstinence syndrome in the infant. We used a threshold of HIV RNA $<75$ copies/ml to define a viral load (VL) as undetectable.

Infants with at least one positive virological marker of infection at any age and/or persistence of antibodies beyond 18 months of age were considered to be HIV-positive, while infants with a negative PCR test result for HIV DNA or RNA and/or negative HIV antibody test were classified as HIV-negative, regardless of age (excluding negative PCR test results on the day of delivery). Infants with conflicting results or without data available were categorised as having an indeterminate HIV status. For multiple births, data on MTCT were based on the first-born infant only unless infection status was discordant between twins/triplets, in which case the infected infant was retained.

Combined antiretroviral therapy (cART) was defined as $\geq 3$ antiretroviral drugs taken simultaneously; 12 women receiving antenatal dual therapy were included in the monotherapy group throughout. Elective caesarean 
section was defined as before rupture of membranes and onset of labour.

\section{Data analysis}

Univariable comparisons were assessed with the chi-square test or Fisher's exact test for categorical variables and the Wilcoxon rank sum test for continuous variables. Poisson regression models with robust variance estimators were fitted to obtain prevalence ratios (PR) and adjusted prevalence ratios (aPR) and $95 \%$ confidence intervals (CI) in analyses investigating factors associated with positive HSV-2 serostatus. Socio-demographic characteristics and co-infections associated with HSV-2 seropositivity in univariable analyses $(p<0.1)$ were included in the multivariable model, with the exception of those which were considered likely to have post-dated HSV-2 seroconversion.

Logistic regression models fitted to investigate the impact of HSV-2 seropositivity on risk of HIV MTCT were adjusted a priori for factors previously found to be associated with HIV MTCT in this cohort (use of antenatal ART, mode of delivery, preterm delivery and IDU history) [13] and time period of delivery (2007-09 vs 2010-12) and centre, which was included as a random effect to account for regional differences in clinical practice and in maternal characteristics. Sub-analyses were conducted to explore the association between HSV-2 seropositivity and HIV MTCT among (a) mother-child pairs with maternal syphilis serology, as positive syphilis serology has previously been associated with HIV MTCT risk in this population [19]; (b) women who delivered vaginally; (c) women who did not receive antenatal cART.

Statistical analysis was performed using STATA version 12 (Stata Corp LP, College Station USA).

\section{Results}

The 1513 HIV-positive women with HSV-2 serostatus available were enrolled into the postnatal cohort a median of 3.9 months (interquartile range (IQR) 1.1-10.3) after delivery. Median maternal age was 27.3 years (IQR 24.1-30.8) and $87 \%(1306 / 1509)$ were married or cohabiting. Just under half $(647 / 1423)$ were primiparous, and $53 \%(796 / 1513)$ had been diagnosed as HIVinfected during their most recent pregnancy, with 143 (9\%) diagnosed in the third trimester or during delivery. A fifth (295/1504) of women had an IDU history and a similar proportion $(286 / 1464)$ had a sexual partner who injected drugs.

Median antenatal CD4 count measurement was 430 cells $/ \mathrm{mm}^{3}$ (IQR 290-580), measured at median 21.3 weeks gestation (IQR 15.6-26.7); $36 \%(414 / 1165)$ had a CD4 count $\leq 350$ cells $/ \mathrm{mm}^{3}$ and $15 \%(204 / 1321)$ had WHO Stage 3 or 4 HIV disease. Almost all $(96 \%, 1451 / 1509)$ received antenatal ART but most discontinued this at delivery, with only a quarter (410/1498) still on treatment at postnatal cohort enrolment. Only $53 \%$ (800/1513) of women had an antenatal HIV RNA measurement; 373 women had a measurement available within 90 days (median 37 days) before delivery and while on ART, of whom $42 \%$ (158) were virologically suppressed after a median of 9.7 weeks (IQR 5.3-13.4) on treatment.

HSV-2 seroprevalence was $68 \%(1026 / 1513)$; for $87 \%$ (1309/1509), the HSV-2 antibody test was conducted during pregnancy, for $6 \%(88 / 1509)$ prior to conception and $7 \%(112 / 1509)$ after delivery. Table 1 presents maternal and delivery characteristics stratified by HSV-2 serostatus.

\section{Factors associated with positive HSV-2 seropositivity}

The proportion of women with HSV-2 antibodies increased significantly with age, from $57 \%$ of women aged $16-23$ years to $71 \%$ of those aged $>30$ years, with higher parity and history of pregnancy termination also associated with increased probability of positive HSV-2 serostatus (Table 1). With respect to other STIs, $1.9 \%$ (9/ 481) of HSV-2 seronegative women and $2.0 \%(20 / 981)$ of HSV-2 seropositive women had positive serology for syphilis (Fisher's exact test $p=1.0), 10.6 \%(43 / 405)$ and $31 \%(158 / 511)$ respectively had a positive chlamydia test $\left(x^{2}=54.37, p<0.01\right)$ and $0.5 \%(2 / 430)$ and $0.3 \%(2 / 759)$ respectively for gonorrhoea (Fisher's exact test $p=0.62$ ). Women with HSV-2 antibodies were more likely to have a history of IDU, be positive for HCV antibodies and HBsAg, and report an HIV-positive partner (Table 1). HSV-2 seropositive women were more likely to report their most recent pregnancy as having been unplanned (Table 1, $p=0.06$ ), but reported better access to family planning than HSV-2 seronegative women at cohort enrolment (Table 1).

A greater proportion of women with HSV-2 antibodies had been diagnosed with HIV before conception (Table 1) and $8 \%(81 / 973)$ conceived on ART compared with $4 \%$ $(18 / 466)$ of seronegative women $\left(\chi^{2}=9.79, p<0.01\right)$. Among those initiating ART during pregnancy, HSV-2 seropositive women were more likely to receive cART than seronegative women (51 \% (451/892) vs. $37 \%(165 /$ 448) respectively, $\left.\chi^{2}=22.64, p<0.01\right)$ and a slightly higher proportion had a pre-ART CD4 count of $\leq 350$ cells $/ \mathrm{mm}^{3}$ ( $38 \%\left(249 / 649\right.$ vs. $\left.32 \%(56 / 176), \chi^{2}=2.55, p=0.11\right)$. Of 455 women who had a viral load measure before ART start (373 (82 \%) of whom were HSV-2 seropositive), median viral load was 11369 copies/ml (IQR 2392, 38676), with no difference by HSV-2 serostatus (Wilcoxon rank sum test $p=0.76)$. Overall $16 \%(154 / 934)$ of HSV-2 seropositive women had WHO stage 3 or 4 disease compared with $13 \%(50 / 387)$ of HSV-2 seronegative women $\left(\chi^{2}=2.67, p=0.10\right)$.

The multivariable model exploring factors associated with HSV-2 seropositivity excluded access to family 
Table 1 Maternal socio-demographic and clinical characteristics, stratified by HSV-2 status

\begin{tabular}{|c|c|c|c|}
\hline Characteristic & $\begin{array}{l}\text { HSV-2 seronegative } \\
n(\%)\end{array}$ & $\begin{array}{l}\text { HSV-2 seropositive } \\
n(\%)\end{array}$ & $x^{2}$ value and $p$ value \\
\hline Maternal age $(n=1512)$ & & & $x^{2}=20.34 p<0.001$ \\
\hline $16-23$ years & $116(24)$ & $155(15)$ & \\
\hline 24-26 years & $148(30)$ & $302(29)$ & \\
\hline $27-30$ years & $119(24)$ & $319(31)$ & \\
\hline 31 years & $104(21)$ & $249(24)$ & \\
\hline Marital status $(n=1509)$ & & & $x^{2}=0.82 p=0.364$ \\
\hline Married/cohabiting & $415(85)$ & $891(87)$ & \\
\hline Single/divorced/widowed & $71(15)$ & $132(13)$ & \\
\hline Age at leaving full-time education $(n=1088)$ & & & $x^{2}=4.39 p=0.111$ \\
\hline$\leq 16$ years & $53(21)$ & $222(27)$ & \\
\hline $17-18$ years & $65(26)$ & $230(27)$ & \\
\hline$\geq 19$ years & $133(53)$ & $385(46)$ & \\
\hline Parity $(n=1423)$ & & & $x^{2}=16.70 p<0.001$ \\
\hline 0 & $245(53)$ & $402(42)$ & \\
\hline 1 & $165(36)$ & $392(41)$ & \\
\hline$\geq 2$ & $54(12)$ & $165(17)$ & \\
\hline Previous pregnancy terminations $(n=1398)$ & & & $x^{2}=63.35 p<0.001$ \\
\hline 0 & $280(62)$ & $373(40)$ & \\
\hline 1 & $79(17)$ & $214(23)$ & \\
\hline$\geq 2$ & $95(21)$ & $357(38)$ & \\
\hline Pregnancy planned $(n=1501)$ & & & $x^{2}=3.44 p=0.064$ \\
\hline No & $130(27)$ & $320(31)$ & \\
\hline Yes & $355(73)$ & $696(69)$ & \\
\hline Has access to family planning $(n=1087)$ & & & $x^{2}=28.02 p<0.001$ \\
\hline No & $75(16)$ & $39(6)$ & \\
\hline Yes & $388(84)$ & $585(94)$ & \\
\hline Timing of HIV diagnosis $(n=1513)$, & & & $x^{2}=6.06 p=0.109$ \\
\hline Before pregnancy & $167(34)$ & $407(40)$ & \\
\hline First or second trimester & $264(54)$ & $532(52)$ & \\
\hline Third trimester/delivery & $56(12)$ & $87(8)$ & \\
\hline History of IDU $(n=1504)$ & & & $x^{2}=6.48 p=0.011$ \\
\hline No & $409(84)$ & $800(79)$ & \\
\hline Yes & $77(16)$ & $218(21)$ & \\
\hline Partner's HIV status ${ }^{\mathrm{a}}(n=1352)$ & & & $x^{2}=36.95 p<0.001$ \\
\hline Unknown & $137(32)$ & $238(26)$ & \\
\hline Negative & $144(34)$ & $210(23)$ & \\
\hline Positive & $146(34)$ & $477(52)$ & \\
\hline HCV serostatus $(n=1402)$ & & & $x^{2}=46.82 p<0.001$ \\
\hline Negative & $344(79)$ & $589(61)$ & \\
\hline Positive & $89(21)$ & $380(39)$ & \\
\hline Hepatitis B surface antigen ( $n=1451)$ & & & $x^{2}=18.89 p<0.001$ \\
\hline Negative & $409(87)$ & $756(77)$ & \\
\hline
\end{tabular}


Table 1 Maternal socio-demographic and clinical characteristics, stratified by HSV-2 status (Continued)

\begin{tabular}{|c|c|c|c|}
\hline Positive & $62(13)$ & $224(23)$ & \\
\hline Antenatal/intrapartum antiretrovirals $(n=1508)$ & & & $x^{2}=31.42 p<0.001$ \\
\hline CART & $183(38)$ & $542(53)$ & \\
\hline ZDV +/- sdNVP & $283(58)$ & $443(43)$ & \\
\hline None or sdNVP only & $19(4)$ & $38(4)$ & \\
\hline
\end{tabular}

${ }^{\mathrm{a}}$ As reported by the woman

planning, because the higher levels of access reported by HSV-2 seropositive women may have been due to treatment-seeking for symptomatic STIs (including genital ulcer disease) or termination of pregnancy and therefore may post-date HSV-2 seroconversion. Chlamydia was also excluded because, as an often transient bacterial infection, it is likely to have been acquired in the period immediately preceding testing and after chronic HSV-2 infection. In the final adjusted model, women with a history of pregnancy termination (vs no history), who reported their partner to be HIV-positive (vs of unknown HIV status) and who had $\mathrm{HCV}$ antibodies remained at increased risk of HSV-2 seropositivity (Table 2).

Although IDU history was associated with HSV-2 seropositivity among 1402 women in univariable analysis, this association was not apparent among the 1095 women included in the adjusted model (Table 2), indicating some bias in the complete-case analysis. However, among 1394 women with both IDU history and HCV serostatus available, the association between HSV-2 antibodies and HCV seropositivity after adjusting for IDU history remained (APR $1.3195 \%$ CI 1.21-1.42) with no association between HSV-2 antibodies and IDU after adjusting for HCV antibodies (APR $0.9595 \% \mathrm{CI} 0.86-1.03$ ), indicating that the observed association between HCV and HSV-2 seropositivity was not explained by factors specific to IDUs (e.g. sexual risk behaviours). Of note, $22 \%(249 / 1119)$ of women without an IDU history in this population had HCV antibodies vs $79 \%(217 / 275)$ of those with an IDU history.

\section{Infants and MTCT}

Delivery and infant data are presented in Table 3. Infants of HSV-2 seropositive mothers were more likely to be delivered vaginally and to be of low birthweight than other infants (Table 3). Almost all (99.4 \%, 1497/1506) infants were exclusively formula fed. At the time of analyses, all children were at least 2 years of age (median 4.98 years, IQR 3.76-6.26) and DNA PCR test results were available for $80 \%(1216 / 1513)$. HIV status was available for 89 and $83 \%$ of infants of HSV-2 seropositive and -negative women respectively $\left(\chi^{2}=11.86, p\right.$ $<0.01)$; 37 infants were HIV-infected, giving an overall HIV MTCT rate of $2.8 \%$ (95 \% CI 1.98-3.84) (Table 3). There was no evidence that HSV-2 seropositivity was associated with HIV MTCT risk in unadjusted analyses
(Tables 3 and 4, HIV MTCT rate was $3.2 \%$ among HSV-2 seronegative women and $2.6 \%$ among HSV-2 seropositive women, $X^{2}=0.37, p=0.54$ ).

In multivariable analyses, the only factor associated with HIV MTCT was lack of antenatal ART, associated with a three-fold increased risk (Table 4). In the first of the three sub-analyses of factors associated with HIV MTCT risk, adjusting additionally for maternal syphilis serology among 1241 mother-child pairs with this available, the AOR for positive versus negative HSV-2 serostatus was 1.39 (95\% CI $0.51-3.80, p=0.523$ ). In the sub-analysis restricted to the 927 women delivering vaginally the AOR for HSV-2 seropositive vs seronegative was 1.46 (95\% CI 0.40-5.34 $p=0.565)$ and in the subanalysis restricted to 629 women without antenatal cART, the AOR was also not significantly increased (AOR 1.57, 95 \% CI 0.30-8.12, $p=0.590$ ).

\section{Discussion}

In this large cohort of HIV-positive pregnant women, we found that two-thirds had antibodies to HSV-2. Almost all women received antenatal ART and around half received cART; the HIV MTCT rate was $2.8 \%$ overall, with no increased risk of MTCT of HIV detected among HSV-2 seropositive women.

Recent global estimates of prevalent HSV-2 in adults aged 15-49 years in 2012 indicated prevalence in females of $14.8 \%$ and in males of $8.0 \%$; in Europe the estimate was $10 \%$ for women and $4 \%$ for men [20]. High HSV-2 prevalence is reported in women living with HIV, for example, $86 \%$ of HIV-positive pregnant women in a Nairobi study [21]. In a Canadian study of Africa/Caribbean-born women, prevalence of HSV-2 infection was $86 \%$ in HIV-positive and $47 \%$ in HIV-negative women [22]. Thus our finding of a seroprevalence of $68 \%$ is consistent with published estimates.

HSV-2 is a persistent infection and thus prevalence increases with age, as seen here and demonstrated elsewhere. In the global estimates study, prevalence increased from $<5 \%$ in $15-19$ year olds, to $13 \%$ by age $30-34$ and $17 \%$ by age $45-49$ [20]. In our adjusted analyses we found that HSV-2 seropositive women were more likely to have a history of pregnancy termination, which may be a marker of greater sexual risk, including lack of or inconsistent use of barrier contraception. In this population, 
Table 2 Factors associated with a maternal positive HSV-2 serostatus

\begin{tabular}{|c|c|c|c|c|c|}
\hline & $\begin{array}{l}\text { Proportion of women } \\
\text { with HSV-2 antibodies }\end{array}$ & PR $(95 \% \mathrm{Cl})$ & $p$ value & $\begin{array}{l}\text { Adjusted PR (95 \% Cl) } \\
n=1095\end{array}$ & $p$ value \\
\hline \multicolumn{6}{|l|}{ Maternal age } \\
\hline $16-23$ years & $57 \%(155 / 271)$ & 1.00 & & 1.00 & \\
\hline $24-26$ years & $67 \%(302 / 450)$ & $1.19(1.04-1.37)$ & 0.014 & $1.11(0.97-1.28)$ & 0.137 \\
\hline $27-30$ years & $73 \%$ (319/438) & $1.25(1.09-1.44)$ & 0.002 & $1.09(0.95-1.26)$ & 0.218 \\
\hline$\geq 31$ years & $71 \%(249 / 353)$ & $1.23(1.07-1.43)$ & 0.004 & $1.03(0.88-1.21)$ & 0.676 \\
\hline \multicolumn{6}{|l|}{ Parity } \\
\hline 0 & $62 \%(402 / 647)$ & 1.00 & & 1.00 & \\
\hline 1 & $70 \%(392 / 557)$ & $1.13(1.03-1.23)$ & 0.009 & $1.01(0.91-1.10)$ & 0.913 \\
\hline$\geq 2$ & $75 \%(165 / 219)$ & $1.14(1.01-1.28)$ & 0.027 & $0.99(0.87-1.12)$ & 0.857 \\
\hline \multicolumn{6}{|c|}{$\begin{array}{l}\text { History of pregnancy } \\
\text { termination }\end{array}$} \\
\hline 0 & $57 \%(373 / 653)$ & 1.00 & & 1.00 & \\
\hline 1 & $73 \%(214 / 293)$ & $1.24(1.11-1.38)$ & $<0.001$ & $1.22(1.10-1.36)$ & $<0.001$ \\
\hline$\geq 2$ & $79 \%(357 / 452)$ & $1.36(1.25-1.49)$ & $<0.001$ & $1.30(1.18-1.43)$ & $<0.001$ \\
\hline \multicolumn{6}{|c|}{ Pregnancy planned } \\
\hline No & $71 \%(320 / 450)$ & 1.00 & & 1.00 & \\
\hline Yes & $66 \%(696 / 1051)$ & $0.96(0.88-1.05)$ & 0.343 & $1.03(0.94-1.13)$ & \\
\hline \multicolumn{6}{|l|}{ History of IDU } \\
\hline No & 66 \% (800/1209) & 1.00 & & 1.00 & \\
\hline Yes & 74 \% (218/295) & $1.05(0.96-1.16)$ & 0.299 & $0.93(0.83-1.03)$ & 0.174 \\
\hline \multicolumn{6}{|c|}{ Partner's HIV status ${ }^{\mathrm{a}}$} \\
\hline Unknown & $63 \%(238 / 375)$ & 1.00 & & 1.00 & \\
\hline Negative & $59 \%(210 / 354)$ & $0.90(0.79-1.04)$ & 0.147 & $0.91(0.79-1.05)$ & 0.184 \\
\hline Positive & $77 \%$ (477/623) & $1.21(1.10-1.33)$ & $<0.001$ & $1.15(1.05-1.26)$ & 0.003 \\
\hline \multicolumn{6}{|l|}{ HCV serostatus } \\
\hline Negative & $63 \%(589 / 933)$ & 1.00 & & 1.00 & \\
\hline Positive & 81 \% (380/469) & $1.25(1.16-1.35)$ & $<0.001$ & $1.23(1.13-1.35)$ & $<0.001$ \\
\hline \multicolumn{6}{|c|}{$\begin{array}{l}\text { Hepatitis B surface } \\
\text { antigen }\end{array}$} \\
\hline Negative & $65 \%(756 / 1165)$ & 1.00 & & 1.00 & \\
\hline Positive & $78 \%$ (224/286) & $1.20(1.11-1.31)$ & $<0.001$ & $1.06(0.97-1.16)$ & 0.179 \\
\hline
\end{tabular}

${ }^{\mathrm{a}}$ As reported by the woman

condoms are the main form of contraception [23] which is an ineffective family planning method and of only moderate effectiveness in preventing acquisition of HSV-2 [24]. Other factors associated with significantly greater probability of HSV-2 seropositivity were having a known HIV-positive partner and being co-infected with HCV. The epidemiology of HCV infection in Ukraine is not well understood with over a fifth of non-IDUs in this cohort reported to be HCV seropositive. HCV is more sexually transmissible among HIV-positive individuals [25] and our findings of an association between HCV and HSV-2 seropositivity may indicate shared risk factors for sexual acquisition. However, risk factors for $\mathrm{HCV}$ acquisition in this setting may also include iatrogenic exposures, which could have been more common among HSV-2 seropositive women given their greater likelihood of history of pregnancy termination.

We did not detect an association between HSV-2 antibodies and increased risk of HIV MTCT; this is in contrast with findings from other studies, which reported an association between HSV-2 antibodies or genital ulcer disease and HIV MTCT [15, 16, 26, 27]. These studies were all conducted more than 10 years ago in populations with much higher background rates of HIV MTCT. Our sample size of 404 HSV-2 seronegative and 916 seropositive mothers and $3.2 \%$ transmission rate in the seronegative group allowed us to rule out a 2.25 -fold increased risk of HIV MTCT with HSV-2 antibodies (i.e. a transmission rate 
Table 3 Delivery and infant characteristics, stratified by maternal HSV-2 serostatus

\begin{tabular}{|c|c|c|c|}
\hline & HSV-2 seronegative $n(\%)$ & HSV-2 seropositive $n(\%)$ & $x^{2}$ value and $p$ value \\
\hline Total live births & 487 & 1026 & - \\
\hline Mode of delivery $(n=1483)$ & & & $x^{2}=73.17 p<0.001$ \\
\hline Vaginal & $277(58)$ & $784(78)$ & \\
\hline Elective CS & $180(38)$ & $176(18)$ & \\
\hline Emergency CS & $20(4)$ & $46(5)$ & \\
\hline Gestational age $(n=1505)$ & & & $x^{2}=0.45 p=0.501$ \\
\hline$\geq 37$ completed weeks & $445(92)$ & $928(91)$ & \\
\hline$<37$ completed weeks & $39(8)$ & $93(9)$ & \\
\hline \multicolumn{4}{|l|}{ Birth weight $(n=1508)$} \\
\hline$\geq 2500 \mathrm{~g}$ & $441(91)$ & $890(87)$ & $x^{2}=4.25 p=0.039$ \\
\hline$<2500 \mathrm{~g}$ & $45(9)$ & $132(13)$ & \\
\hline HIV infection status $(n=1513)$ & & & $x^{2}=12.22 p<0.01$ \\
\hline Infected & $13(2.67)$ & $24(2.34)$ & \\
\hline Uninfected & $391(80.29)$ & $892(86.94)$ & \\
\hline Indeterminate/unknown & $83(17.04)$ & $110(10.72)$ & \\
\hline MTCT rate (\%) where infant & $3.2 \%(95 \%$ Cl 1.7-5.4) & $2.6 \%(95 \%$ Cl 1.7-3.9) & $x^{2}=0.37, p=0.54$ \\
\hline HIV status known $(n=1320)$ & & & \\
\hline
\end{tabular}

of $\geq 7.2 \%$ ), with $80 \%$ power and alpha 0.05 . To have detected a $50 \%$ increased risk, as previously reported in the Zimbabwean study [27], would have required a much larger sample size of around $1850 \mathrm{HSV}-2$ seronegative and 4200 HSV-2 seropositive women, given the low HIV MTCT rate overall. Therefore, although we did not detect an association between HSV-2 antibodies and increased risk of HIV MTCT, our study did not have sufficient statistical power to rule out the smaller increases in HIV MTCT risk associated with HSV-2 seropositivity found previously, due to the low HIV MTCT rates overall in our study population, almost all of whom received antenatal ART.

Although we showed a significant association between MTCT and use of ART, we were unable to explore the association between HSV-2 serostatus and MTCT after adjusting for maternal HIV viral load because of limited viral load testing: of the 37 HIVinfected infants, 23 were born to women with no antenatal HIV RNA measure available and only five mothers of the remaining 14 had a measurement available in the 90 days preceding delivery. The HIV MTCT rate reported here was substantially lower than that observed in the Ukraine ECS overall in the same time period (4.56\%, $95 \%$ CI 4.04-5.13), because our analysis was restricted to a postnatal cohort who generally had better access to PMTCT interventions, reflecting greater engagement with antenatal and HIV care services [13].

The high prevalence of HSV-2 found in our HIVpositive pregnant women has implications for their infants. Neonatal HSV-2 infection is a serious condition associated with high morbidity and mortality, with antiviral prophylaxis for HSV-2 recommended during pregnancy in women with frequent clinical recurrences. In HIV co-infected women this could have the additional benefit of reducing HIV viral load and/or HIV MTCT risk. However, risk of vertical transmission of HSV-2 is higher in primary infection during pregnancy (around $40 \%)$ compared to recurrent infections (1-3\%) [28]. PCR test results and data on clinical symptoms were not available, and we were therefore unable to make conclusions about the timing of HSV-2 seroconversions here or prevalence of genital ulcer disease during pregnancy, however most HSV-2 seropositive women had been tested during pregnancy (87\%) or pre-conception (6\%). Of HSV-2 seronegative women, 1 in 10 had a recent chlamydia diagnosis, indicating ongoing sexual risk and potential risk of HSV-2 acquisition during pregnancy. Up to $20 \%$ of HSV-2 seronegative women with an $\mathrm{HSV}$-2-infected partner will seroconvert during pregnancy, with highest risk of neonatal infection if this occurs late in pregnancy [29]. Some experts suggest partner testing of pregnant women susceptible to HSV2 and counselling of those with a positive partner about abstinence and reducing oral-genital contact near term [30].

As the postnatal cohort enrols women retained in HIV care following delivery, our study population may have had better uptake of sexual and reproductive health services. Our results may therefore not be generalizable to the entire HIV-positive childbearing population in Ukraine; in reality, HSV-2 seroprevalence 
Table 4 Factors associated with MTCT of HIV

\begin{tabular}{|c|c|c|c|}
\hline & Number with MTCT (\%) & Odds ratio $(95 \% \mathrm{Cl})$ & $\begin{array}{l}\text { Adjusted odds ratio }(95 \% \mathrm{Cl}) \text {, } \\
p \text { value } n=1286^{\mathrm{a}}\end{array}$ \\
\hline \multicolumn{4}{|l|}{ Length of gestation } \\
\hline$\geq 37$ completed weeks & $33 / 1204(2.7)$ & 1 & 1 \\
\hline$<37$ completed weeks & 4/109 (3.7) & $0.73(0.25-2.09) p=0.553$ & $0.93(0.30-2.82) p=0.891$ \\
\hline \multicolumn{4}{|l|}{ Mode of delivery } \\
\hline Vaginal & 27/942 (2.9) & 1 & 1 \\
\hline Elective CS & 9/305 (3.0) & $1.03(0.48-2.21) p=0.945$ & $0.76(0.32-1.80) p=0.529$ \\
\hline Emergency CS & $1 / 58(1.7)$ & $0.58(0.08-4.38) p=0.602$ & $1.04(0.13-8.28) p=0.973$ \\
\hline \multicolumn{4}{|l|}{ Antenatal/intrapartum ARVs } \\
\hline CART & $11 / 607(1.8)$ & 1 & 1 \\
\hline ZDV +/- sdNVP & 20/666 (3.0) & $0.61(0.29-1.28) p=0.190$ & $0.59(0.27-1.31) p=0.194$ \\
\hline None or sdNVP only & 6/42 (14.3) & $5.31(2.01-14.03) p<0.01$ & $3.42(1.19-9.85) p=0.023$ \\
\hline \multicolumn{4}{|l|}{ IDU history } \\
\hline No & 30/1057 (2.8) & 1 & 1 \\
\hline Yes & $7 / 254(2.8)$ & $0.98(0.43-2.26) p=0.964$ & $1.18(0.46-2.99) p=0.735$ \\
\hline \multicolumn{4}{|l|}{ HSV-2 serostatus } \\
\hline Negative & 13/404 (3.2) & 1 & 1 \\
\hline Positive & 24/916 (2.6) & $0.80(0.41-1.60) p=0.553$ & $1.43(0.54-3.77) p=0.474$ \\
\hline
\end{tabular}

${ }^{a}$ Adjusted additionally for time period of delivery (2007-09 vs. 2010-12) and including random effect term for centre of enrolment

may be higher if women not engaged with services after delivery were also more likely to have HSV-2 coinfection. More research is needed on occurrence of genital ulcer disease among HSV-2 seropositive women and outcomes of their neonates, and more generally about sexual health of women in Ukraine living with or at high risk of acquiring HIV, to strengthen preventative health services

\section{Conclusion}

In conclusion, we found a high prevalence of HSV-2 antibodies but no associated increased risk of vertical HIV transmission, in this population with an overall HIV MTCT rate of $2.8 \%$. High prevalence of recent chlamydia diagnosis indicated ongoing sexual risk, suggesting that HSV-2 seronegative women may benefit from counselling on prevention of HSV-2 acquisition during pregnancy and that clinicians should be alert to the possibility of a primary maternal infection, while HSV-2 seropositive women with clinical symptoms require appropriate obstetric management.

\section{Declarations}

\section{Ethics approval and consent to participate}

The Women's Study is nested within the ECS, which has ethical approval from the Great Ormond Street Hospital for Children NHS Trust/Institute of Child Health Research Ethics Committee (reference 96EB02). Local approvals were also obtained from all the participating HIV/AIDS Centres.
Women were required to give verbal consent to take part in the ECS and to complete an anonymised questionnaire at enrolment into the Women's Study; the return of this questionnaire was taken as documentation and evidence of consent to participate in the Women's Study.

\section{Consent for publication \\ Not applicable.}

\section{Availability of data and materials}

As our dataset contains potentially patient-identifiable data and data items on sensitive health information such as HIV infection status, other sexually transmitted infections and substance use, our data are not automatically available for sharing. We provide pseudonymised data sets for inclusion in pooled analyses and systematic reviews. We invite people who are interested in accessing our data to contact Claire Thorne for further information.

\footnotetext{
Abbreviations

HSV 2: Herpes simplex virus type 2; HIV: human immunodeficiency virus; MTCT: mother to child transmission; STI: sexually transmitted infection; ECS: European Collaborative Study; PMTCT: prevention of MTCT; VL: viral load; IDU: injecting drug use; CART: combined antiretroviral therapy; PCR: polymerase chain reaction; DNA: deoxyribonucleic acid; RNA: ribonucleic acid; HCV: hepatitis C virus; PR: prevalence ratio; aPR: adjusted prevalence ratio; $\mathrm{Cl}$ : confidence Interval; IQR: interquartile range; HbsAg: hepatitis $B$ surface antigen.
}

Competing interests

There were no conflicts of interest in regard to this manuscript. 
KAP has received travel grants from AbbVie and Bristol Meyers Sqibb. CT has received funding from the UK Medical Research Council, Public Health England, UNICEF, the PENTA Foundation and AbbVie. HB has received funding from the UK Medical Research Council, the International AIDS Society and Public Health England.

\section{Authors' contributions}

KAP, HB and CT contributed to study conception, study design, study performance, data analysis, and article writing. KAP, HB, CT, RM, AV contributed to study conception and article review. The Ukraine European Collaborative Study contributed to data collection. All authors were involved in data interpretation and revised the article critically. All authors read and approved the final manuscript.

\section{Acknowledgements}

Ukraine European Collaborative Study: Tatyana Pilipenko (Perinatal Prevention of AIDS Initiative, Odessa, Ukraine); Dr Svetlana Posokhova (Regional Hospital, Odessa, Ukraine); Dr Tatyana Kaleeva, Dr Yulia Barishnikova, Dr Stanislav Servetsky, Dr Rostislav Tereshenko (Odessa Regional Centre for HIV/AIDS, Ukraine); Dr Svetlana Solokha, Dr Nikolay Grazhdanov (Donetsk Regional Centre for HIV/AIDS, Ukraine); Dr Irina Raus, Dr Alexander Yurchenko, Dr Irina Adejnova (Kiev City Centre for HIV/AIDS, Ukraine); Dr Zinaida Ruban, Dr Olena Govorun "Dr Lyudmila Ostrovskaya (Mykolaiv Regional Centre for HIV/AIDS, Ukraine); Dr Natalya Primak; Dr. Liane Kvasha (Kriviy Rig City Center for HIV/AIDS, Ukraine).

\section{Funding}

This project has received funding from the European Union's Seventh Framework Programme for research, technological development and demonstration under EuroCoord grant agreement $n^{\circ} 260694$

\section{Author details}

'Division of Infectious Diseases, University Hospital Bern, CH-3010 Bern, Switzerland. ${ }^{2}$ Population, Policy and Practice Programme, UCL Institute of Child Health, University College London, London, UK. ${ }^{3}$ Perinatal Prevention of AIDS Initiative, Odessa, Ukraine. ${ }^{4}$ Shupyk National Medical Academy of Postgraduate Education, Kiev, Ukraine.

\section{Received: 26 September 2015 Accepted: 21 April 2016}

\section{Published online: 27 April 2016}

\section{References}

1. Ukrainian Center of Social Control Dangerous Diseases, Ministry of Health of Ukraine. HIV Infection in Ukraine, National bulletin No 43. Kiev: 2015.

2. World Bank. Synthesis Report: HIV in the European Region. http:// documents.worldbank.org/curated/en/2013/05/17796656/hiv-europeanregion-using-evidence-strengthen-policy-programmes-vulnerabilityresponse-synthesis-report. Accessed 12 May 2015.

3. Uuskula A, McMahon JM, Raag M, et al. Emergent properties of HIV risk among injection drug users in Tallinn, Estonia: synthesis of individual and neighbourhood-level factors. Sex Transm Infect. 2010;86 Suppl 3:iii79-84.

4. Landes $M$, Thorne C, Barlow $P$, et al. Prevalence of sexually transmitted infections in HIV-1 infected pregnant women in Europe. Eur J Epidemiol. 2007:22(12):925-36.

5. Reinheimer C, Doerr HW. Prevalence of herpes simplex virus type 2 in different risk groups: thirty years after the onset of HIV. Intervirology. 2012;55(6):395-400.

6. Freeman EE, Weiss HA, Glynn JR, et al. Herpes simplex virus 2 infection increases HIV acquisition in men and women: systematic review and meta-analysis of longitudinal studies. AIDS. 2006;20(1):73-83.

7. Gupta R, Warren T, Wald A. Genital herpes. Lancet. 2007;370(9605):2127-37.

8. Aumakhan B, Gange SJ, Beyrer C, et al. Quantitative and qualitative correlates of cervicovaginal herpes simplex virus type 2 shedding among HIV-infected women in the Women's Interagency HIV Study. Int J STD AIDS. 2011;22(5):273-7.

9. Corey L, Ashley R, Valaciclovir HSVTSG. Prevention of herpes simplex virus type 2 transmission with antiviral therapy. Herpes. 2004;11 Suppl 3:170A-4A.

10. Townsend $C L$, Byrne L, Cortina-Borja $M$, et al. Earlier initiation of ART and further decline in mother-to-child HIV transmission rates, 2000-2011. AIDS. 2014;28(7):1049-57.
11. Aebi-Popp K, Mulcahy F, Glass TR, et al. Missed opportunities among HIV-positive women to control viral replication during pregnancy and to have a vaginal delivery. J Acquir Immune Defic Syndr. 2013;64(1):58-65.

12. Thorne C, Semenenko I, Pilipenko T, Ukraine European Collaborative Study G, et al. Progress in prevention of mother-to-child transmission of HIV infection in Ukraine: results from a birth cohort study. BMC Infect Dis. 2009;9:40.

13. Bailey $\mathrm{H}$, Townsend $\mathrm{CL}$, Semenenko I, et al. Impact of expanded access to combination antiretroviral therapy in pregnancy: results from a cohort study in Ukraine. Bull World Health Organ. 2013;91(7):491-500.

14. Bollen $\amalg$, Whitehead SJ, Mock PA, et al. Maternal herpes simplex virus type 2 coinfection increases the risk of perinatal HIV transmission: possibility to further decrease transmission? AIDS. 2008;22(10):1169-76.

15. Chen KT, Segu M, Lumey LH, et al. Genital herpes simplex virus infection and perinatal transmission of human immunodeficiency virus. Obstet Gynecol. 2005;106(6):1341-8.

16. Drake AL, Roxby AC, Ongecha-Owuor F, et al. Valacyclovir suppressive therapy reduces plasma and breast milk HIV-1 RNA levels during pregnancy and postpartum: a randomized trial. J Infect Dis. 2012;205(3):366-75.

17. Perti T, Saracino M, Baeten JM, et al. High-dose valacyclovir decreases plasma HIV-1 RNA more than standard-dose acyclovir in persons coinfected with HIV-1 and HSV-2: a randomized crossover trial. J Acquir Immune Defic Syndr. 2013;63(2):201-8.

18. Thorne C, Semenenko I, Malyuta R. Prevention of mother-to-child transmission of human immunodeficiency virus among pregnant women using injecting drugs in Ukraine, 2000-10. Addiction. 2012:107(1):118-28.

19. Thorne $\mathrm{C}$ et al. Correspondence: Mother-to-Child transmission risk is increased among HIV-infected pregnant women in Ukraine with serological test results positive for syphilis. Clin Infect Dis. 2008;47:1114.

20. Looker KJ, Magaret AS, Turner KM, et al. Global estimates of prevalent and incident herpes simplex virus type 2 infections in 2012. PLoS One. 2015; 10(1):e114989.

21. Roxby AC, Liu AY, Drake AL, et al. Short communication: T cell activation in HIV-1/herpes simplex virus-2-coinfected Kenyan women receiving valacyclovir. AIDS Res Hum Retrovir. 2013;29(1):94-8.

22. Remis RS, Liu J, Loutfy M, et al. The epidemiology of sexually transmitted co-infections in HIV-positive and HIV-negative African-Caribbean women in Toronto. BMC Infect Dis. 2013;13:550.

23. Saxton J, Malyuta R, Semenenko I, et al. Previous reproductive history and post-natal family planning among HIV-infected women in Ukraine. Hum Reprod. 2010;25(9):2366-73.

24. Martin ET, Krantz E, Gottlieb SL, et al. A pooled analysis of the effect of condoms in preventing HSV-2 acquisition. Arch Intern Med. 2009;169(13):1233-40.

25. Tohme RA, Holmberg SD. Is sexual contact a major mode of hepatitis C virus transmission? Hepatology. 2010;52(4):1497-505.

26. Cowan FM, Humphrey JH, Ntozini R, et al. Maternal Herpes simplex virus type 2 infection, syphilis and risk of intra-partum transmission of HIV-1: results of a case control study. AIDS. 2008;22(2):193-201.

27. Drake AL, John-Stewart GC, Wald A, et al. Herpes simplex virus type 2 and risk of intrapartum human immunodeficiency virus transmission. Obstet Gynecol. 2007;109(2 Pt 1):403-9.

28. Brown ZA, Wald A, Morrow RA, et al. Effect of serologic status and cesarean delivery on transmission rates of herpes simplex virus from mother to infant. JAMA. 2003;289(2):203-9.

29. Gardella C, Brown Z, Wald A, et al. Risk factors for herpes simplex virus transmission to pregnant women: a couples study. Am J Obstet Gynecol. 2005;193(6):1891-9.

30. Gardella C, Krantz E, Daruthayan C, et al. The acceptance of HSV-testing partners of HSV-2 seronegative pregnant women. Sex Transm Dis. 2009; 36(4):211-5. 